\title{
Motion correction for diffusion weighted SMS imaging
}

Citation for published version (APA):

Herbst, M., Poser, B. A., Singh, A., Deng, W., Knowles, B., Zaitsev, M., Stenger, V. A., \& Ernst, T. (2017). Motion correction for diffusion weighted SMS imaging. Magnetic Resonance Imaging, 38, 33-38.

https://doi.org/10.1016/j.mri.2016.12.013

Document status and date:

Published: 01/05/2017

DOI:

10.1016/j.mri.2016.12.013

Document Version:

Publisher's PDF, also known as Version of record

Document license:

Taverne

Please check the document version of this publication:

- A submitted manuscript is the version of the article upon submission and before peer-review. There can be important differences between the submitted version and the official published version of record.

People interested in the research are advised to contact the author for the final version of the publication, or visit the DOI to the publisher's website.

- The final author version and the galley proof are versions of the publication after peer review.

- The final published version features the final layout of the paper including the volume, issue and page numbers.

Link to publication

\footnotetext{
General rights rights.

- You may freely distribute the URL identifying the publication in the public portal. please follow below link for the End User Agreement:

www.umlib.nl/taverne-license

Take down policy

If you believe that this document breaches copyright please contact us at:

repository@maastrichtuniversity.nl

providing details and we will investigate your claim.
}

Copyright and moral rights for the publications made accessible in the public portal are retained by the authors and/or other copyright owners and it is a condition of accessing publications that users recognise and abide by the legal requirements associated with these

- Users may download and print one copy of any publication from the public portal for the purpose of private study or research.

- You may not further distribute the material or use it for any profit-making activity or commercial gain

If the publication is distributed under the terms of Article $25 \mathrm{fa}$ of the Dutch Copyright Act, indicated by the "Taverne" license above, 
Technical note

\title{
Motion correction for diffusion weighted SMS imaging
}

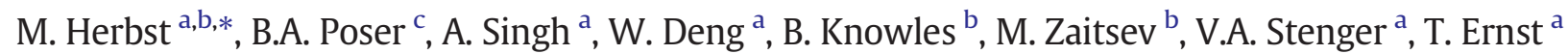 \\ ${ }^{a}$ Department of Medicine, John A. Burns School of Medicine, University of Hawaii, Honolulu, HI, USA \\ ${ }^{\mathrm{b}}$ Department of Radiology, Medical Physics, Medical Center University of Freiburg, Faculty of Medicine, University of Freiburg, Freiburg, Germany \\ ${ }^{c}$ Maastricht Brain Imaging Centre, Faculty of Psychology E' Neuroscience, Maastricht University, Netherlands
}

\section{A R T I C L E I N F O}

\section{Article history:}

Received 15 August 2016

Received in revised form 13 December 2016

Accepted 13 December 2016

\section{Keywords:}

Motion correction

Diffusion weighted imaging

Simultaneous multi-slice

Multiband

\section{Introduction}

Diffusion-weighted imaging (DWI) is commonly used in clinical MRI, in particular in the assessment of stroke, demyelination, or cortical lesions [1,2]. It also plays an important role in scientific studies of structural connectivity [3-5].

Although DWI sequences usually use fast readouts, such as echo-planar imaging (EPI), DWI scans commonly last several minutes, especially when used for diffusion tensor imaging (DTI). These long scans result in sensitivity to head motion [6,7]. Motion correction with external optical tracking can improve DWI data quality [8-11]. However, affixation of tracking markers can be challenging and marker "slippage" during the scan will compromise the quality of motion correction or even introduce artifacts [12,13].

Parallel imaging makes it possible to shorten the EPI readout for diffusion imaging [14,15], and thereby reduce echo time (TE) or increase spatial resolution. However, this does not substantially reduce acquisition times, due to the rather long weighting gradients required for DWI combined with typically extensive slice packages, which results in prolonged repetition times, TR. In contrast, simultaneous multi-slice imaging (SMS) makes it possible to acquire several slices simultaneously [16-19] and can considerably shorten volume acquisition times for DTI protocols. However, this gain in scanning efficiency is often used to acquire more complex DWI protocols (such as multiple b-values,

\footnotetext{
* Corresponding author at: Department of Medicine, John A. Burns School of Medicine, University of Hawaii, Honolulu, HI, USA

E-mail address: michael.herbst@uniklinik-freiburg.de (M. Herbst).
}

more diffusion directions, and higher resolution), reintroducing the problem of patient motion.

Motion correction for SMS-DWI can therefore be expected to improve DWI acquisitions. However, the effect of motion, including prospective motion correction (PMC), on SMS acquisitions and reconstructions, has not been investigated. Therefore, we incorporated prospective motion correction into diffusion weighted SMS imaging and investigated the influence of motion, motion correction, and relative coil motion on the reconstructed data. The algorithm described in [20] was extended to incorporate SMS reconstruction into multiplexed sensitivity encoding (MUSE) [21,22]. Additionally, a technique was implemented to address potential marker slippage during prospective motion correction with external optical tracking, using intrinsic position information of the volumetric data.

\section{Methods}

All experiments were conducted on a 3T Tim Trio system (Siemens Healthcare, Erlangen, Germany), using a 32 channel head-coil. External tracking was performed with an in-bore camera system (Metria Innovation Inc. Milwaukee, USA) [23]. Tracking markers were attached using double sided tape. A single spin-echo EPI sequence with diffusion weighting was modified to allow for continuous real-time position updates [9], SMS acquisition [18,24], and acquisition in either single-shot or segmented mode. A variable number of non-diffusion weighted scans ( $b=0$ images) were distributed evenly over the acquisition. The orientations were defined by the electrostatic repulsion algorithm [25]. Unless otherwise stated, online SMS reconstructions of single- 
shot data were performed using the sliceGRAPPA implementation as distributed with blipped-CAIPI EPI (https://www.nmr.mgh.harvard. edu/software/c2p/sms). In vivo acquisitions were performed in accordance with the local regulations and all subjects provided verbal and written consent using a protocol approved by our Institutional Review Board.

\subsection{Multiplexed sensitivity encoding (MUSE) for segmented acquisitions}

Segmentation of the EPI readout allows for higher resolution and better SNR. However, segmented EPI scans are notoriously sensitive to motion due to phase corruption, requiring adjustment of phase between in the acquired segments of $\mathrm{k}$-space. We used a dedicated reconstruction algorithm (MUSE, [10,21]), based on a two-step SENSE [14] reconstruction. In a first step, each under-sampled segment is reconstructed separately. Phase information from this reconstruction is combined with the coil sensitivity maps, and utilized in a second SENSE reconstruction step that integrates all segments of $\mathrm{k}$-space into a full data set. This technique can be used for single-slice and SMS reconstructions alike [21,22].

This study comprised a set of experiments.

\subsection{PACE-XPACE: integration of image-based and external motion feedback}

The external tracking system provides position information in 6 degrees of freedom ( 3 rotations and 3 translations) which are represented in the affine transformation matrix $A_{\text {Extern }}$ [26]. Independently, the vendor-provided Prospective Acquisition CorrEction (PACE) algorithm yields the transformation matrix $A_{P A C E}$ through real-time volumetric registration to the first scan [27]. However, since the diffusion weighed data required additional offline reconstruction (MUSE), the PACE mechanism was modified to provide feedback only based on the non-diffusion weighted scans. Clearly, unlike the external tracking data, the PACE updates will be available only sparsely, depending on the number of $b=0$ images interspersed into the time series.

If prospective motion correction with external tracking perfectly tracks head motion, then all intrinsic PACE updates would return the identity matrix. Therefore, when external tracking is enabled, the matrix APACE represents an error term, for instance, if the tracking marker shows slippage relative to the brain. Consequently, the position information from the two sources can be combined into a single transformation $A_{\text {final }}$, by multiplying the data from the tracking system $\left(A_{\text {Extern }}\right)$ with the inverse of the PACE feedback $\left(A^{-1}{ }_{\text {PACE }}\right)$ :

$\mathrm{A}_{\text {final }}=\mathrm{A}_{\text {Extern }} * \mathrm{~A}^{-1}$ PACE

Intrinsic motion feedback from the imaging data will therefore allow correction matrix $\left(A_{\text {final }}\right)$ to be "reinitialized" during the course of the scan (after acquisition of each $b=0$ volume).

A phantom experiment was performed to evaluate this approach. A structured phantom was equipped with two tracking markers and placed inside the head-coil. Both targets were visible to the camera.

In a reference experiment, tracking information from a single target was transferred the XPACE motion correction algorithm. During the DWI measurement, the phantom was intentionally moved between $\mathrm{b}=0$ scans and this motion was prospectively corrected using tracking information AXPACE from the first marker.

In a second experiment, external tracking was then manipulated by switching tracking data from the first to the second marker (within a scan), creating intentional errors in motion correction and hence position errors. These errors are detected by the PACE algorithm and the positional information $A_{P A C E}$ is introduced to the final transformation matrix according to Eq. (1).

If a certain selectable threshold (overall rotation or translation) of residual motion $A_{P A C E}$ is exceeded, according to the image-based volumetric registration, then a subset of the measurement is repeated. A subset is defined a set of diffusion weighted images between adjacent $\mathrm{b}=0$ scans. A motion threshold of $1.5 \mathrm{~mm}$ or 1.0 degrees was empirically found to be safely beyond the noise level and provides a good compromise between potential data loss and scan time prolongation. Also, the potential number of repetitions was limited to ensure acceptable scan duration. In the current implementation, this was achieved by specifying a maximal number of rejections. After this number is reached, no further rejection is accepted. However, the measurement will still be motion corrected. Sequence parameters: TR/TE: 4400/ $65 \mathrm{~ms}$, TA: $5 \mathrm{~min}$, resolution: $1.5 \times 1.5 \times 2 \mathrm{~mm}^{3}, 2$ segments, matrix: $128 \times 128$, partial Fourier $=5 / 8,45$ slices, SMS factor 1 , diffusion weightings $b_{1}=500 \mathrm{~s} / \mathrm{mm}^{2}$ with 30 directions and $6 \mathrm{~b}=0$ scans. The phantom was manually rotated after the first $b=0$ volume. While this rotation leads to flow artifacts in our water-filled phantom, the long TR left enough time for any water motion to settle before the next $b=0$ volume.

\subsection{Effect of virtual motion on the coil sensitivities}

Separation of simultaneously acquired slices requires knowledge of the coil sensitivity profiles, either in the form of coil-sensitivities (SENSE) or in a reconstruction Kernel (GRAPPA). Motion changes the relative position between the object and the receiver coils, and therefore may introduce errors into reconstructed images. To investigate the influence of such misalignments on the slice-GRAPPA reconstruction, a phantom experiment was performed using artificial motion feedbacks as described in [28]. This techniques uses position input form a text file to update the acquisition during runtime. This allows for very well defined position changes. Note, that this virtual motion only changes the position of the FoV, and therefore the position relative to the acquired coil sensitivities, while the signal distribution within the object remains unchanged. Several artificial pose changes (2 degrees, $2 \mathrm{~mm}$, and 10 degrees in plane) were generated between $b=0$ volumes after the slice-GRAPPA kernel was computed at the initial object position.

During the course of the measurement, the position offset is registered by the volumetric PACE motion algorithm and fed back to the scanner as $A_{\text {PACE}}$, to test the PACE functionality during SMS acquisitions. The volumetric data was used to correct for the artificial position change and re-establish conformity with the slice-GRAPPA kernel.

Sequence parameters: TR/TE: 1500/60 ms, TA: $0.5 \mathrm{~min}$, resolution: $2 \times 2 \times 3 \mathrm{~mm}^{3}$, 1 segment, SMS factor 3, matrix: $96 \times 96,45$ slices, diffusion weightings $\mathrm{b}_{1}=500 \mathrm{~s} / \mathrm{mm}^{2}$ with 12 directions and $5 \mathrm{~b}=0$ scans.

\subsection{Augmented MUSE: post-hoc adjustment of coil sensitivities in case of} real motion

Augmented SENSE [20] describes a theoretical framework which allows initial coil sensitivity data to be adapted to motion-induced changes in object poses with regards to RF coils. It assumes precise knowledge of the coils sensitivities, even somewhat beyond the boundaries of the measured object.

We extended the augmented SENSE framework to MUSE reconstructions (hence "augmented MUSE"), by adapting the coil sensitivities for the two MUSE reconstruction steps described above.

In the first step, the image of one segment is reconstructed using augmented SENSE:

$\mathrm{s}_{1}(\mathrm{t})=\int C^{\prime}(\boldsymbol{r}) \rho_{1}(r) \mathrm{e}^{-\mathrm{i} \mathrm{k}_{1}(\mathrm{t}) \boldsymbol{r}} \mathrm{d} \boldsymbol{r}$

where $s_{1}(t)$ is the acquired signal from one segment and $k_{1}(t)$ is the generalized 3D SMS k-space as described in [29]. $C^{\prime}(\boldsymbol{r})$ is the receiver 
coil sensitivity $C(\boldsymbol{r})$ which is modified with the transformation matrix from the external tracking data:

$C^{\prime}(\boldsymbol{r})=C(\boldsymbol{r}) * \mathrm{~A}_{\mathrm{extern}}$

As described in [30], the general MUSE formulation can be written as a system of equations for $\mathrm{L}$ acquired segments:

$$
\begin{aligned}
\mathrm{s}_{1}(\mathrm{t}) & =\int\left[\mathrm{C}^{\prime}(\mathbf{r}) \mathrm{e}^{i \varphi_{1}(\mathbf{r})}\right] \rho(\mathbf{r}) \mathrm{e}^{-i k_{1}(\mathrm{t}) \mathbf{r}} \mathrm{d} \mathbf{r} \\
& \vdots \\
\mathrm{s}_{\mathrm{L}}(\mathrm{t}) & =\int\left[\mathrm{C}^{\prime}(\mathbf{r}) \mathrm{e}^{i \varphi_{\mathrm{L}}(\mathbf{r})}\right] \rho(\mathbf{r}) \mathrm{e}^{-i k_{\mathrm{L}}(\mathrm{t}) \mathbf{r}} \mathrm{d} \mathbf{r}
\end{aligned}
$$

where $\varphi_{1 . . L}$ are the image phases calculated from the undersampled data of each segment (Eq. (2)) which are now multiplied with the modified coilsensitivities $C^{\prime}(\boldsymbol{r})$ (Eq. (3)).

The impact of motion versus coil sensitivities was first investigated in a phantom experiment. During a DWI measurement, the phantom was moved multiple times, primarily by rotational movements (up to 20 degrees). This motion was corrected prospectively using XPACE tracking. Offline SENSE reconstruction was then performed first without modifications to the coil sensitivity maps, and then using maps adjusted for the corrected motion.

Sequence parameters: TR/TE: 1700/55 ms, resolution: $1.5 \times 1.5 \times 2 \mathrm{~mm}^{3}$, SMS factor 3, 2 segments, matrix: $128 \times 128,45$ slices, diffusion weightings $\mathrm{b}_{1}=200 \mathrm{~s} / \mathrm{mm}^{2}$ with 30 directions and 6 $\mathrm{b}=0$ scans.

Next, the augmented MUSE experiment was repeated in vivo. A volunteer was instructed to move as much as the head-coil allows. This resulted in motion patterns with amplitudes of $10 \mathrm{~mm}$ and 5 degrees. Three in vivo acquisitions were performed: (a) No motion (for reference), (b) motion without correction and (c) motion combined with prospective correction. Data from the motion-corrected experiment (c) were reconstructed twice, using regular SMS-MUSE with unmodified coil sensitivities, and using augmented MUSE reconstruction.

Sequence parameters for the in vivo scans were: TR/TE: 2000/80 ms, resolution: $1.5 \mathrm{~mm}$ isotropic, SMS factor 3, 2 segments, matrix: $128 \times 128$, 45 slices, diffusion weightings $b_{1}=1000 \mathrm{~s} / \mathrm{mm}^{2}$ with 30 directions and $6 \mathrm{~b}=0$ scans.

\section{Results}

The effectiveness of the PACE-XPACE combination is shown in Fig. 1. Panels a-d display one slice of the $b=0$ images collected at four time points during the phantom experiment. Panel (a) shows the phantom in the original position. In (b) the phantom was moved manually, but the movement was prospectively corrected using the external tracking system. The movement of the small air bubble at the top of the phantom reflects the changing orientation. The third time-point ( $1 \mathrm{c}$ and $\mathrm{c}^{*}$ ) shows images acquired after switching tracking to the second marker, which introduces a position offset (c) that is then detected and retrospectively corrected by re-alignment with PACE $\left(c^{*}\right)$. Panel $(d)$ shows the reacquisition of volume $\mathrm{c}$ after the prospective correction data was adapted to the intentionally modified marker position (as per Eq. (1)).

Fig. 2 shows the effects of motion on the validity of the coil sensitivity maps in a phantom. Tracking data from a text file were used to introduce a defined artificial movement. The first column (a) shows the phantom in its original position at the beginning of each measurement. In the second column (b) three in-plane movements were generated: 10 degrees rotation (first row), 2 degrees rotation (second row), and $2 \mathrm{~mm}$ translation (third row). These results show that only large movements (10 degrees) appear to have a substantial influence on the reconstruction (b, top row, pointed out by the arrow). In panel c, these artificial movements were then detected and corrected by the volumetric registration in PACE followed by the acquisition of a new data set. No residual artifacts are seen when the artificial motion was corrected (c), in accordance with expectations for virtual motion.

Fig. 3 shows an average of all images acquired during the prospective motion correction experiment without (a) and with (b) additional adaption of the coil sensitivities using augmented MUSE. For each case, three simultaneously acquired slices are displayed. Comparing the two images (Fig. 3, a and b), it can be seen that adjusting coil sensitivities improves reconstruction (as pointed out by the arrow). However, some remaining artifacts can still be seen using augmented MUSE (b).

The results of the in vivo experiments are summarized in Fig. 4. Images were acquired without motion (a), with motion but no PMC (b), with motion and prospective correction (c), and motion with PMC + augmented MUSE (d). The motion was up to approximately $10 \mathrm{~mm}$ translation and 5 degrees rotation, and similar across acquisitions. Motion markedly reduces the image quality compared to the nomotion case (b versus a). Prospective motion correction substantially improves results (c versus b). In the in vivo study, the use of augmented MUSE did not visibly improve the data (d versus $\mathrm{c}$ ), despite the relatively

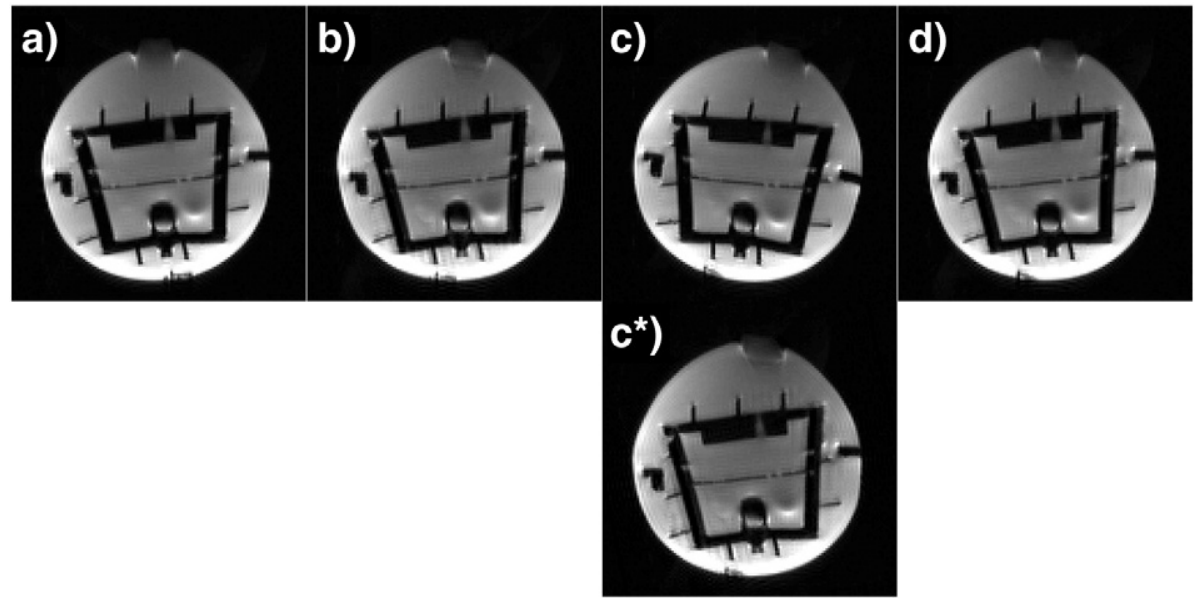

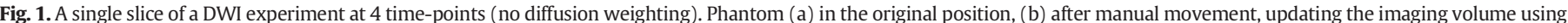

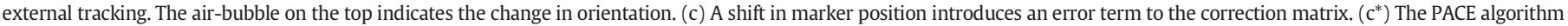

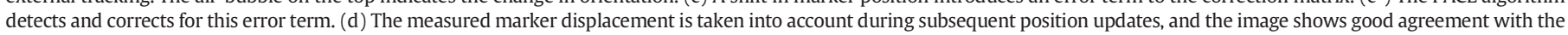
original position shown in panel (a). 


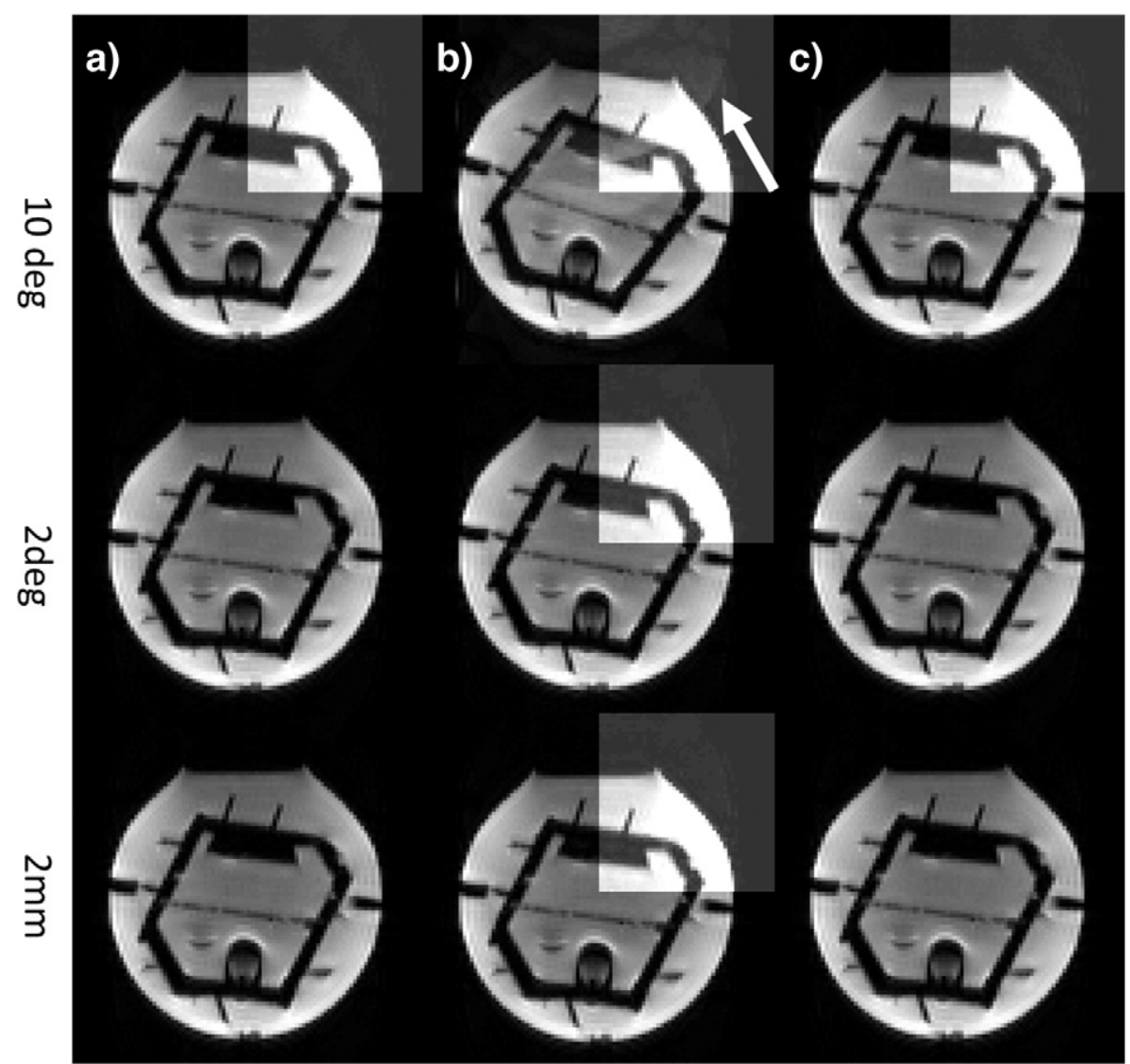

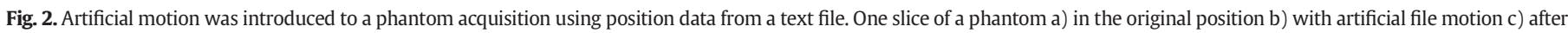

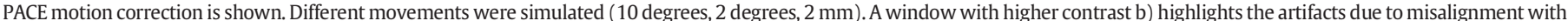
the coil sensitivities.

large motion. When motion corrected data ( $\mathrm{c}$ and $\mathrm{d}$ ) is compared to the 'no motion' case (a) a slight blurring can be observed after motion correction due to residual misalignment of the imaging data.

\section{Discussion}

This work investigated the influence of motion and its correction on SMS imaging. We investigated the use of prospective motion correction with external tracking, the use of volumetric navigators using intrinsic positional information, and a combination of both approaches. Additionally, the influence of inconsistencies between coil and imaging data on the SMS reconstruction was investigated. We have shown, following the theoretical framework shown in [20], that coil sensitivities can be adapted to motion while having only minor influence on the presented vivo DTI data.

The use of prospective motion correction with external tracking is advantageous and allows for extremely fast position updates [8-10,23, $26,30]$. However, instabilities due to incorrect motion correction have

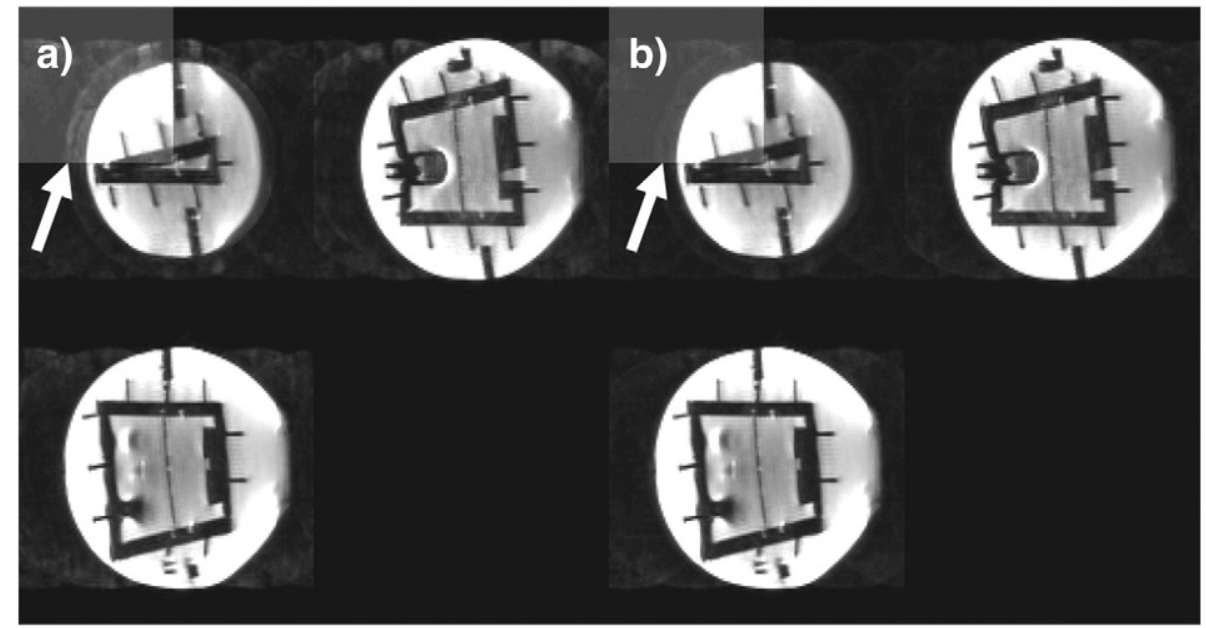

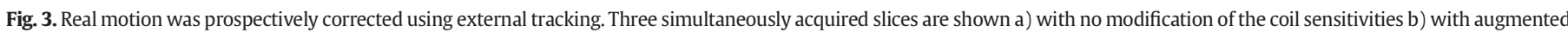
MUSE. In the upper left corner, the contrast was enhanced to show remaining artifacts. 


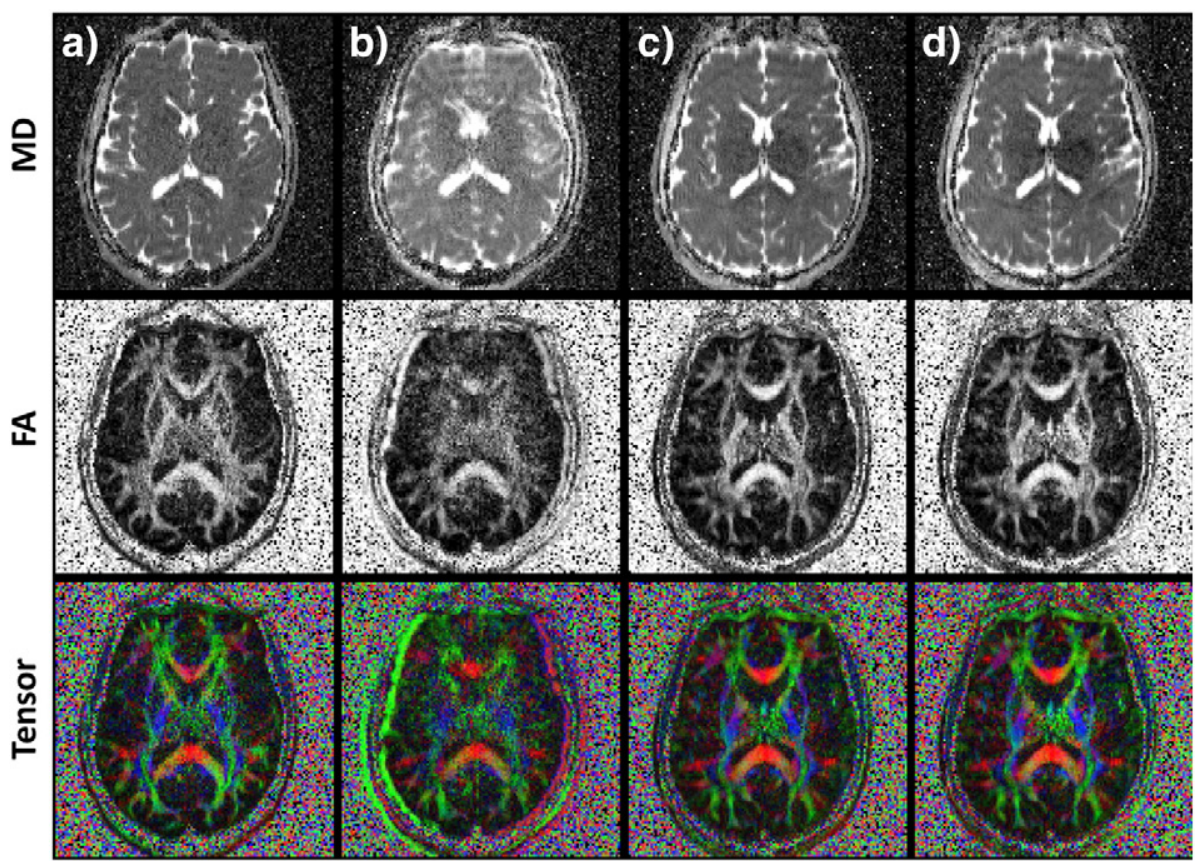

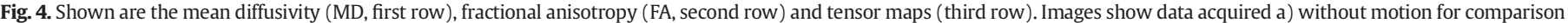
b) with motion, without correction c) with prospectively corrected motion. Additionally, the third dataset was reconstructed using augmented MUSE d).

also been reported $[12,13]$. The combination of external tracking data with image-based navigator information can help overcome this problem. While external tracking can provide information at high rates (30-100 Hz), the navigator data delivers occasional updates that allow a recalibration of the correction matrix of the external tracking system. While this work uses PACE as intrinsic tracking method [27], which is particularly appropriate for fMRI and DTI acquisitions, other navigator approaches [31-35] could be incorporated and make the method available to other imaging or spectroscopy techniques. The current implementation was restricted to segmented and SMS acquisitions. However, it should be feasible to extend the technique to more complex acquisition schemes.

Since parallel imaging relies on the correct information to reconstruct non-acquired data points (SENSE: sensitivity maps; GRAPPA: kernel), the effect of motion and its correction need to be evaluated. Therefore, we investigated the effect of motion on single-shot SMS as well as on segmented EPI acquisitions, which involve additional inplane under-sampling in the first reconstruction step. Our experiments show that small movements (up to about $2 \mathrm{~mm}$ and 2 degrees) have only minor effects on parallel imaging reconstructions and can be neglected in practice. It is to be noted, that the virtual motion experiment only reproduces some relevant motion effects and the artifact behavior in case of the true motion may be enhanced by the additional phenomena, such as coil loading or susceptibility-induced field changes.

For larger movements ( $>10$ degrees), ghosting-like residual aliasing can be seen even after motion correction. We demonstrated an improvement in image quality with Augmented MUSE in a phantom experiment. Crucially, Augmented MUSE relies on the knowledge of coil sensitivities outside the object, obtained by extrapolation of the sensitivities. In practice, large movements may violate this requirement. While it might be possible to demonstrate these effects on selected in vivo raw images, no visible influence was observed on the processed DTI data, even in the case of extreme movements. To the contrary, our results indicate that the effect of motion-induced changes in coil sensitivities may be negligible, especially for motions that are typically encountered, or accepted, in practice. These observations might be specific to the coil setup used in this study, and motion in a receiver array with smaller elements (where the same motion would yield larger relative changes in sensitivities) may have more substantial effects. In addition, the purely qualitative investigation of a single subject was not sufficient to investigate potential changes in DTI data. However, recent studies report similar findings and state notable effects in BOLD fMRI with parallel imaging for rather large head motions in the range of $5 \mathrm{~mm}$ and 5degrees [36,37]. In the future, Augmented MUSE for DTI will be included in studies with larger cohorts to investigate quantitative effects on the DTI data.

In summary, we investigated the benefits of image-based and external motion correction on simultaneous-multislice diffusion-weighted acquisitions at 3T. Integrating optical and image based motion correction effectively removed errors related to slippage of the optical marker, which is one of the challenges for external motion tracking $[12,13]$. Furthermore, motion-induced inconsistencies in coil sensitivity profiles resulted in small but noticeable errors in SMS reconstruction of phantom data. We introduced augmented MUSE to improve segmented EPI reconstructions, but observed only negligible qualitative differences in the in vivo dataset investigated in this work.

\section{Acknowledgements}

This work was supported by NIH R01DA019912, R01EB011517, and K02DA02056. Michael Herbst was supported by the Alexander von Humboldt Foundation.

\section{References}

[1] Acosta-Cabronero J, Nestor PJ. Diffusion tensor imaging in Alzheimer's disease: insights into the limbic-diencephalic network and methodological considerations. Front Aging Neurosci Oct. 2014;6.

[2] Conturo TE, McKinstry RC, Aronovitz JA, Neil JJ. Diffusion MRI: precision, accuracy and flow effects. NMR Biomed 1995;8(7):307-32.

[3] Bammer R, Holdsworth SJ, Veldhuis WB, Skare ST. New methods in diffusionweighted and diffusion tensor imaging. Magn Reson Imaging Clin N Am May 2009;17(2):175-204

[4] Basser PJ, Jones DK. Diffusion-tensor MRI: theory, experimental design and data analysis - a technical review. NMR Biomed Dec. 2002;15(7-8):456-67.

[5] Filler A. MR neurography and diffusion tensor imaging: origins, history \&amp; clinical impact. Nat Preced Apr. 2009.

[6] Anderson AW, Gore JC. Analysis and correction of motion artifacts in diffusion weighted imaging. Magn Reson Med 1994;32(3):379-87.

[7] Le Bihan D, Poupon C, Amadon A, Lethimonnier F. Artifacts and pitfalls in diffusion MRI. J Magn Reson Imaging Sep. 2006;24(3):478-88. 
[8] Aksoy M, et al. Real-time optical motion correction for diffusion tensor imaging. Magn Reson Med Aug. 2011;66(2):366-78.

[9] Herbst M, Maclaren J, Weigel M, Korvink J, Hennig J, Zaitsev M. Prospective motion correction with continuous gradient updates in diffusion weighted imaging. Magn Reson Med 2012;67(2):326-38.

[10] Herbst M, Zahneisen B, Knowles B, Zaitsev M, Ernst T. Prospective motion correction of segmented diffusion weighted EPI. Magn Reson Med Dec. 2015;74(6):1675-81.

[11] Gumus K, et al. Prevention of motion-induced signal loss in diffusion-weighted echo-planar imaging by dynamic restoration of gradient moments: prevention of motion-induced signal loss in DWI. Magn Reson Med Jun. 2014;71(6):2006-13.

[12] Pannetier NA, et al. Quantitative framework for prospective motion correction evaluation. Magn Reson Med Feb. 2016;75(2):810-6.

[13] Singh A, et al. Optical tracking with two markers for robust prospective motion correction for brain imaging. Magn Reson Mater Phys Biol Med Dec. 2015;28(6): 523-34.

[14] Pruessmann KP, Weiger M, Scheidegger MB, Boesiger P, et al. SENSE: sensitivity encoding for fast MRI. Magn Reson Med 1999;42(5):952-62.

[15] Griswold MA, et al. Generalized autocalibrating partially parallel acquisitions (GRAPPA). Magn Reson Med Jun. 2002;47(6):1202-10.

[16] Feinberg DA, et al. Multiplexed Echo Planar Imaging for Sub-Second Whole Brain FMRI and Fast Diffusion Imaging. PLoS ONE Dec. 2010;5(12):e15710.

[17] Larkman DJ, Hajnal JV, Herlihy AH, Coutts GA, Young IR, Ehnholm G. Use of multicoil arrays for separation of signal from multiple slices simultaneously excited. J Magn Reson Imaging 2001;13(2):313-7.

[18] Setsompop K, et al. Improving diffusion MRI using simultaneous multi-slice echo planar imaging. NeuroImage Oct. 2012;63(1):569-80.

[19] Barth M, Breuer F, Koopmans PJ, Norris DG, Poser BA. Simultaneous multislice (SMS) imaging techniques. Magn Reson Med Jan. 2016;75(1):63-81.

[20] Bammer R, Aksoy M, Liu C. Augmented generalized SENSE reconstruction to correct for rigid body motion. Magn Reson Med Jan. 2007;57(1):90-102.

[21] Chen N, Guidon A, Chang H-C, Song AW. A robust multi-shot scan strategy for highresolution diffusion weighted MRI enabled by multiplexed sensitivity-encoding (MUSE). NeuroImage May 2013;72:41-7.

[22] Chang H-C, Guhaniyogi S, Chen N. Interleaved diffusion-weighted improved by adaptive partial-Fourier and multiband multiplexed sensitivity-encoding reconstruction: reconstruction framework for artifact-free DWI. Magn Reson Med May 2015;73(5):1872-84.

[23] Maclaren J, et al. Measurement and Correction of Microscopic Head Motion during Magnetic Resonance Imaging of the Brain. PLoS ONE Nov. 2012;7(11):e48088.
[24] Setsompop K, Gagoski BA, Polimeni JR, Witzel T, Wedeen VJ, Wald LL. Blipped-controlled aliasing in parallel imaging for simultaneous multislice echo planar imaging with reduced g-factor penalty. Magn Reson Med May 2012;67(5):1210-24.

[25] Jones DK, Horsfield MA, Simmons A. Optimal strategies for measuring diffusion in anisotropic systems by magnetic resonance imaging. Magn Reson Med 1999;42.

[26] Zaitsev M, Dold C, Sakas G, Hennig J, Speck O. Magnetic resonance imaging of freely moving objects: prospective real-time motion correction using an external optical motion tracking system. Neurolmage Jul. 2006;31(3):1038-50.

[27] Thesen S, Heid O, Mueller E, Schad LR. Prospective acquisition correction for head motion with image-based tracking for real-time fMRI. Magn Reson Med Sep. 2000; 44(3):457-65.

[28] Herbst M, et al. Reproduction of motion artifacts for performance analysis of prospective motion correction in MRI. Magn Reson Med Jan. 2014;71(1):182-90.

[29] Zahneisen B, Poser BA, Ernst T, Stenger VA. Three-dimensional Fourier encoding of simultaneously excited slices: generalized acquisition and reconstruction framework: 3D Fourier encoding for SMS acquisitions. Magn Reson Med Jun. 2014; 71(6):2071-81.

[30] Herbst M, Deng W, Ernst T, Stenger VA. Segmented simultaneous multi-slice diffusion weighted imaging with generalized trajectories. Magn Reson Med Nov. 2016 epub.

[31] Maclaren J, Herbst M, Speck O, Zaitsev M. Prospective motion correction in brain imaging: a review. Magn Reson Med Mar. 2013:69(3):621-36.

[32] Kober T, Marques JP, Gruetter R, Krueger G. Head motion detection using FID navigators. Magn Reson Med Jul. 2011;66(1):135-43.

[33] Babayeva M, et al. Accuracy and precision of head motion information in multichannel free induction decay navigators for magnetic resonance imaging. IEEE Trans Med Imaging Sep. 2015;34(9):1879-89.

[34] Alhamud A, Tisdall MD, Hess AT, Hasan KM, Meintjes EM, van der Kouwe AJW. Volumetric navigators for real-time motion correction in diffusion tensor imaging. Magn Reson Med Oct. 2012;68(4):1097-108.

[35] Tisdall MD, Hess AT, Reuter M, Meintjes EM, Fischl B, van der Kouwe AJW. Volumetric navigators for prospective motion correction and selective reacquisition in neuroanatomical MRI. Magn Reson Med Aug. 2012;68(2):389-99.

[36] Faraji-Dana Z, Tam F, Chen JJ, Graham SJ. A robust method for suppressing motioninduced coil sensitivity variations during prospective correction of head motion in fMRI. Magn Reson Imaging Oct. 2016;34(8):1206-19.

[37] Faraji-Dana Z, Tam F, Chen JJ, Graham SJ. Interactions between head motion and coil sensitivity in accelerated fMRI. J Neurosci Methods Sep. 2016;270:46-60. 